\title{
New advanced tools for processing and analysis of 2D X-ray diffraction data: XRD2DScan software 7.0
}

\author{
Alejandro Rodriguez-Navarro ${ }^{1}$, Natalia Dadivanyan ${ }^{2}$, Anil Kumar ${ }^{2}$, Milen Gateshki ${ }^{2}$ \\ ${ }^{1}$ Universidad de Granada, 18002 Granada, Spain; \\ ${ }^{2}$ Malvern Panalytical B.V., 7602 EA Almelo, The Netherlands; \\ anava@ugr.es
}

The properties and behaviour of materials (metals, alloys, semiconductors, ceramics, polymers, drugs, biomaterials) are to a large extent determined by their phase composition, particle size, crystallinity, stress, defects and crystallographic orientation (texture). Two-dimensional (2D) X-ray diffraction is one of the most appealing techniques for users who are interested in extracting every bit of information about their samples. 2D X-ray diffraction patterns, collected using area detectors contain detailed information about all these important material characteristics. Furthermore, the high sensitivity and resolution of modern detectors (e.g. PIXcel ${ }^{3 \mathrm{D}}$, GaliPIX ${ }^{3 \mathrm{D}}$ ) make possible the collection of relevant structural information within seconds. This allows following in real time transformation processes of materials, like recrystallization, deformation or phase transitions. XRD2DScan is the Malvern Panalytical software for displaying, processing, and analyzing 2D X-ray diffraction data. The latest version of the software (version 7.0) offers new features such as orientation and crystallite size analysis, image comparison, as well as scripting for easy automation. The application of the software to the characterization of complex anisotropic materials (liquid crystals, polymers, bone, wood, ...) will be illustrated through several examples.

Keywords: 2D X-ray diffraction, microstructure, crystallite size, texture, bone 